\section{Embedded ranging system in ISM band}

X. Yin, J. Bauwelinck, G. Torfs, P. Demuytere,

J. Vandewege, H. Tubbax, J. Wouters, P. Debacker,

J. Olbrechts, P. Spiessens, F. Stubbe and J. Danneels

A new embedded ranging system in the ISM band is described. The proposed indoor ranging system combines the advantages of both broadband and narrowband signals to achieve high ranging accuracy in the presence of strong multipath reflections to achieve a good link budget and to be compliant with spectral regulations. The system is based on a WLAN transceiver (with embedded ranging specific circuits) and uses the same frequency band for both communication and ranging purposes so that no separate dedicated ranging transceiver is needed. A prototype has been implemented to evaluate the performance of the proposed technique in a realistic environment Experimental results show ranging accuracies of less than $20 \mathrm{~cm}$

Introduction: Well-established technologies for position determination mostly address outdoor scenarios, e.g. the Global Positioning System (GPS). While these techniques have adequate performance outdoors and when a sufficiently strong line-of-sight signal is available, they suffer from low accuracy in indoor environments. The key reason for this performance degradation is that the received signals consist of multiple superimposed attenuated, delayed and phase rotated copies of this signal [1]. Especially owing to the narrowband nature of the transmitted signals, these copies have a wide span in the time-domain and hence tend to overlap, as shown in Fig. 1. As a result, copies having travelled several metres more can typically not be separated from the line-of-sight path by the receiver, and it induces errors in the position estimate of the same order of magnitude.

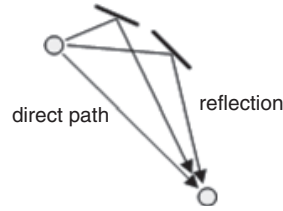

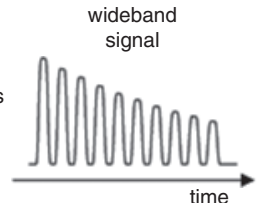

$b$

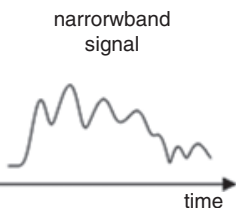

Fig. 1 Transmission over fading multipath channels, and received wideband and narrowband signal in dense multipath environments

$a$ Transmission over fading multipath channels

$b$ Received wideband and narrowband signal in dense multipath environments

To enable accurate position determination in indoor environments, techniques based on received-signal strength (RSS) [2] and propagation-time of signalling $[1,3]$ have been proposed. RSS systems are based on sophisticated pathloss models for indoor environments. But there is no strong correlation between RSS and distance and its distribution depends on hardware implementations [2]. Reference [3] proposed to measure the propagation delay of WLAN packets, but the achieved accuracy $(8 \mathrm{~m})$ is not good enough for most indoor location applications. The propagation time based UWB system provides superior distance estimation accuracy because the transmitted signals feature a very short span in the time-domain and hence provide improved resolution for separating the line-of-sight propagated copy from the reflected copies [1]. However, UWB signalling has serious disadvantages with respect to narrowband communication in terms of the link budget. Because of this, and also the high frequency at which ultra-wideband communication usually takes place, the operational range of such systems tend to be significantly shorter than that of classical narrowband systems. In [4], technologies combining the advantages of narrowband and wideband signals are proposed. In these systems, a narrowband transceiver is used for low-rate data communication, again typically for discovery purposes, whereas a wideband transceiver is used for ranging. The separate ranging transceiver increases system costs and frequency usage is not efficient because the signalling happens in distinct frequency bands.

In this Letter, an indoor ranging scheme is proposed, combining the advantages of both wideband and narrowband signals, which involves a single radio transceiver (with the addition of a few ranging specific circuits) and a single frequency band for both communication and ranging purposes (patented [5]). The fundamental idea is that also relatively narrowband radios such as those used in WLAN products exhibit and/or can be stimulated to generate wideband transient signals, which can then be leveraged for improved positioning accuracy at the receiver, by processing these wideband transient signals rather than just the narrowband communication signal. A proof-of-concept prototype ranging system $(2.4 \mathrm{GHz}$ ISM band) has been developed using commercial off-the-shelf components in order to evaluate the performance of the proposed technique.

Transceiver architecture: Fig. 2 shows the architecture of the WLAN transceiver with embedded ranging. The transceiver is designed for using the existing WLAN infrastructure with minor modifications. While signals with standard channel bandwidth (e.g. $20 \mathrm{MHz}$ ) are used for data communication and nodes discovery, ranging signals having a bandwidth encompassing the whole WLAN band (ISM or U-NII band) can be generated for increasing ranging accuracy. In the transmit path, an RF switch has been inserted between the WLAN transmitter and the power amplifier, which are usually two separate chips. In this way, broadband signals with short rise/fall times can be generated. As typical for WLAN receivers, the receiver implements a direct conversion architecture, but the baseband paths need sufficient bandwidth (e.g. $200 \mathrm{MHz}$ ) to pass the fast rising edges of the RF ranging signals. When a ranging event occurs, the transmitter ranging engine will generate a ranging pulse with short rise/fall time to the RF switch and save the current ranging event timestamp. The received ranging RF pulse will be converted to baseband by using quadrature mixing. Then sampled I and $\mathrm{Q}$ signals are fed into the receiver ranging engine for distance estimation, where the baseband ranging pulse is recovered and the time of arrival (TOA) of the direct path is extracted from the rising edge of the recovered pulse.

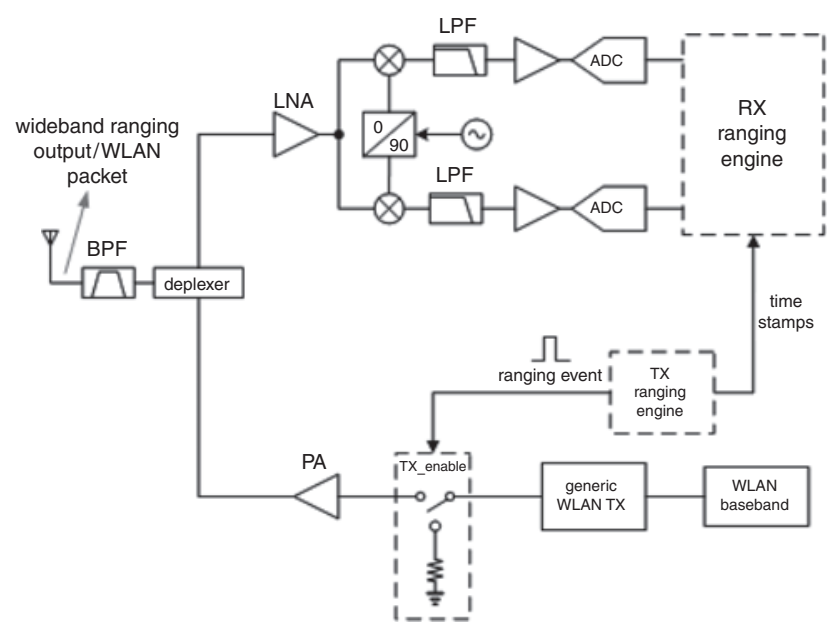

Fig. 2 Conceptual block diagram of proposed ISM band ranging transceiver
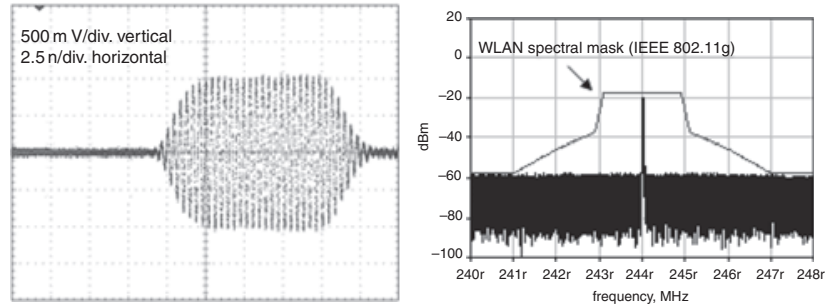

Fig. 3 Transmitter output ranging waveform and spectrum

$a$ Waveform

$b$ Spectrum

Experimental results: The output RF waveform and spectrum are shown in Fig. 3. The output signal spectrum is fully compliant with the WLAN spectral mask (IEEE $802.11 \mathrm{~g}$ ) as long as the ranging events occur at a reasonable rate, e.g. every $40 \mu \mathrm{s}$ (corresponding to 25000 ranging measurements per second). The received signal is shown in Fig. 4, illustrating the relatively strong multipath reflections in the measurement room. In these experiments, the synchronisation between the ranging transmitter and receiver is realised with a coaxial cable of known delay which transfers a trigger signal from transmitter 
to receiver. However, in a practical application, the synchronisation of the different nodes is avoided by performing round-trip time (RTT) measurements.

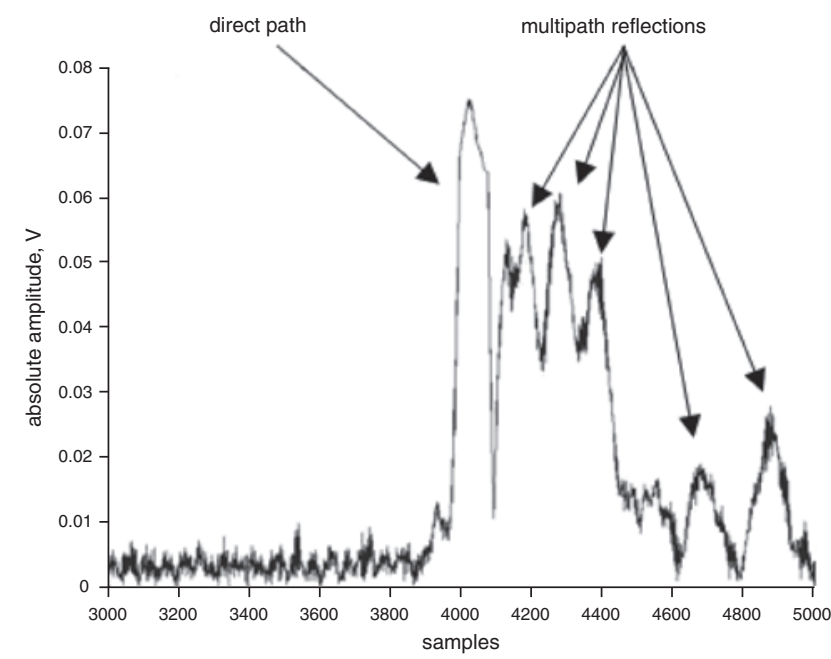

Fig. 4 Received baseband ranging pulse

Several distance measurements are performed out at real distances from 0 to $14.4 \mathrm{~m}$ at intervals of $1.8 \mathrm{~m}$. For each distance, 500 waveforms were statistically analysed. In Fig. 5, the results at $7.2 \mathrm{~m}$ are shown as an example. Both empirical histogram and normal fit illustrate very small absolute error and variance, showing good accuracy of our ranging system. The ranging results are shown in Table 1 and the average of measured data is selected as the statistical estimator for real distance. For all cases a very high accuracy with an error of less than $20 \mathrm{~cm}$ is achieved and the statistical variation is also very small $(3 \sigma<0.5 \mathrm{~m})$.

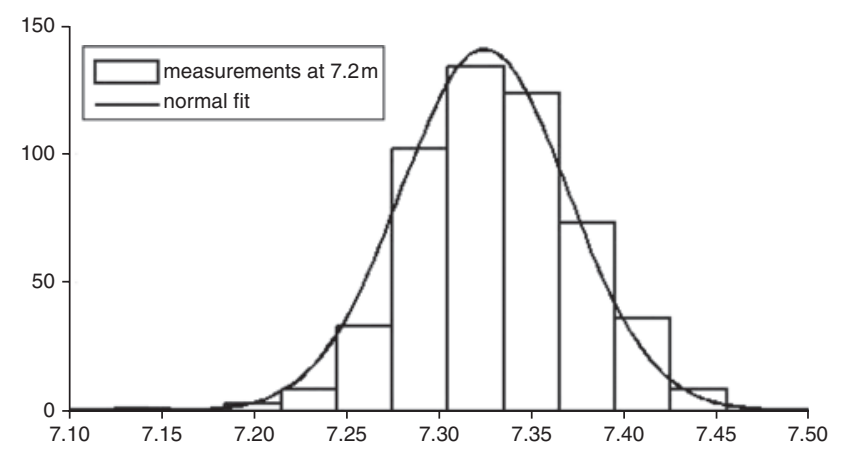

Fig. 5 Histogram and normal fit of measured data at real distance of $7.2 \mathrm{~m}$
Table 1: Ranging results

\begin{tabular}{|c|c|c|c|}
\hline $\begin{array}{c}\text { Distance } \\
(\mathrm{m})\end{array}$ & $\begin{array}{c}\text { Estimated } \\
\text { distance }(\mathrm{m})\end{array}$ & $\begin{array}{c}\text { Standard } \\
\text { deviation }(\mathrm{m})\end{array}$ & $\begin{array}{c}\text { Absolute } \\
\text { error }(\mathrm{m})\end{array}$ \\
\hline 1.8 & 1.937 & 0.029 & 0.14 \\
\hline 3.6 & 3.793 & 0.042 & 0.19 \\
\hline 5.4 & 5.511 & 0.064 & 0.11 \\
\hline 7.2 & 7.324 & 0.045 & 0.12 \\
\hline 9.0 & 8.956 & 0.127 & -0.04 \\
\hline 10.8 & 10.687 & 0.147 & -0.11 \\
\hline 12.6 & 12.656 & 0.079 & 0.06 \\
\hline 14.4 & 14.411 & 0.115 & 0.01 \\
\hline
\end{tabular}

Conclusion: A new embedded ranging system in the ISM band is proposed. The concept does not require a separate dedicated transceiver and is fully compliant with the spectral requirements, even though it uses both broadband and narrowband signals. Experimental results in a multipath environment show that a very high accuracy can be achieved.

Acknowledgment: The authors thank the IWT Vlaanderen for financial support under the scope of the project ELOCA.

(C) The Institution of Engineering and Technology 2008

23 April 2008

Electronics Letters online no: 20081157

doi: 10.1049/el:20081157

X. Yin, J. Bauwelinck, G. Torfs, P. Demuytere and J. Vandewege (INTEC/IMEC, Ghent University, Sint-Pietersnieuwstraat 41, Ghent B-9000, Belgium)

E-mail: xin.yin@intec.ugent.be

H. Tubbax, J. Wouters, P. Debacker, J. Olbrechts, P. Spiessens, F. Stubbe and J. Danneels (Essensium, Gaston Geenslaan 9, Leuven B-3001, Belgium)

\section{References}

1 Gezici, S., Tian, Z., Giannakis, G., Kobayashi, H., Molisch, A., Poor, V., and Sahinoglu, Z.: 'Localization via ultra-wideband radios', IEEE Signal Process. Mag., 2005, 22, (4), pp. 70-84

2 Kaemarungsi, K.: 'Distribution of WLAN received signal strength indication for indoor location determination'. 2006 1st Int. Symp. on Wireless Pervasive Computing, Phuket, Thailand, January 2006

3 Günther, A., and Hoene, C.: 'Measuring round trip times to determine the distance between WLAN nodes', Networking, 2005, pp. 768-779

4 Werb, J.P.: 'Hybrid real time locating system and methodology', International Patent Application, WO 02/088776 A2, May 2002

5 Vandenameele, P.: 'Method for estimating distance between transmitter and receiver, and transmitter and receiver implementing same', International Patent no. WO2007071748 A1, June 2007 